\title{
Síndrome de Burnout em enfermagem pediátrica e neonatal: revisão da literatura
}

Recebido em: 20/03/2013

Aprovado em: 16/05/2014
Renata Santos Tito ${ }^{1}$

Patrícia Campos Pavan Baptista

Fábio José da Silva ${ }^{3}$

Marissol Bastos de Carvalho ${ }^{4}$

Silmar Maria da Silva

Resumo: A síndrome de burnout é caracterizada pelo esgotamento físico e emocional do trabalhador. Foi realizado levantamento da literatura dos últimos 5 anos sobre esta síndrome em enfermagem pediátrica e neonatal. Optou-se pela revisão integrativa da literatura nas bases de Dados: Medline, Cinahl, Lilacs, Dedalus e Scopus. A amostra dessa revisão foi constituída por 13 estudos, dos quais emergiram três categorias temáticas: sofrimento do profissional, características individuais e suporte Institucional. Os dados revelam necessidade de realização de estudos neste campo de atuação com fortes níveis de evidência para subsidiar a saúde do trabalhador de enfermagem.

Descritores: Enfermagem, Enfermagem Pediátrica, Enfermagem Neonatal, Esgotamento Profissional.

Burnout syndrome in pediatric and neonatal nursing: a literature review

Abstract: Burnout syndrome is characterized by physical and emotional exhaustion of workers. We performed a literature of the last 5 years about this syndrome in pediatric and neonatal nursing. We opted for an integrative literature review of data bases: Medline, Cinahl, Lilacs, Dedalus and Scopus. The sample of this review consisted of 13 studies, which revealed three themes: Suffering of the workers, individual characteristics and Institutional Support. The data show the need for studies in this field of work with strong levels of evidence to support worker health nursing.

Descriptors: Nursing, Pediatric Nursing, Neonatal Nursing, Burnout, Professional.

\section{El síndrome de burnout en enfermagem pediatrica y neonatal: una revisión de la literatura}

Resumen: El síndrome de Burnout se caracteriza por agotamiento físico y emocional de los trabajadores. Se realizó un levantamiento de la literatura de los últimos 5 años sobre éste síndrome en enfermería pediátrica y neonatal. Se optó por una revisión integradora de la literatura de las bases de datos: Medline, CINAHL, LILACS, Dedalus y Scopus. La muestra consistió en 13 estudios, de los cuales surgieron tres categorías temáticas: sufrimiento del profesional, características individuales y apoyo Institucional. Los datos muestran la necesidad de realizar estudios con fuertes niveles de evidencia en éste campo para apoyar la salud del trabajador de enfermería.

Descriptores: Enfermería, Enfermería Pediátrica, Enfermería Neonatal, Agotamiento Profesional.

\section{INTRODUÇÃO}

A síndrome de burnout, definida por estresse crônico decorrente do trabalho, caracteriza-se pelo esgotamento físico e emocional do trabalhador, comprometendo seu desempenho nas dimensões profissional, familiar e social. Como consequência, pode ocorrer abandono da profissão, uma vez que o trabalhador não encontra mais sentido da sua relação com o trabalho ${ }^{(1-3)}$.

A equipe de enfermagem, devido ao maior contato com pacientes e familiares dentro do ambiente de trabalho, constituem um grupo com grande predisposição ao desenvolvimento da síndrome de burnout ${ }^{(4-5)}$.

Diante desse conceito, são enumerados vários aspectos do trabalho que tornam a enfermagem uma ocupação vulnerável ao burnout. Essa síndrome além de apresentar implicações relevantes à saúde desses profissionais, compromete a segurança dos pacientes por eles assistidos ${ }^{(6)}$.

As relações entre condições de trabalho e qualidade de vida dos médicos e enfermeiros intensivistas pediátricos e neonatais, avaliada em um estudo acerca do estresse ocupacional, mostram-se prejudicadas. Diante disso e do risco de uma relação menos humana entre o profissional e seu paciente, considera-se necessário reconhecer os estressores presentes no trabalho, além da realização de estudos que avaliem as condições de trabalho e suas repercussões sobre a saúde mental e qualidade de vida dos profissionais envolvidos na assistência ${ }^{(7-9)}$.

Considerando a contribuição para o planejamento de melhorias referentes ao ambiente de trabalho dos profissionais de enfermagem, este artigo objetiva: 1 - Identificar a produção científica sobre a síndrome de burnout em trabalhadores de enfermagem que assistem crianças; 2 - Analisar as evidências científicas sobre a síndrome de burnout em trabalhadores de enfermagem que assistem crianças; 3 - Descrever os fatores relacionados à ocorrência de síndrome de burnout em trabalhadores de enfermagem que assistem crianças;

\section{METODOLOGIA}

Optou-se pela revisão integrativa da literatura, fundamentada em estudos anteriores e definida como método em que conclusões de estudos anteriores são sumarizadas, a fim de que se formulem inferências sobre um tópico específico ${ }^{(10-12)}$. Foi utilizada essa metodologia considerando as 6 etapas seguintes: identificação do tema e seleção da questão de pesquisa, estabelecimento de critérios para inclusão e exclusão de estudos (seleção da amostra), definição

'Enfermeira. Aluna de Mestrado do Programa de Pós-Graduação em Gerenciamento em Enfermagem da EEUSP. E-mail: rstito@usp.br

${ }^{2}$ Enfermeira. Prof. a Doutora da Escola de Enfermagem da Universidade de São Paulo.

${ }^{3}$ Enfermeiro. Aluno de doutorado do Programa de Pós-Graduação em Gerenciamento em Enfermagem da EEUSP.

${ }^{4}$ Enfermeira. Aluna de doutorado do Programa de Pós-Graduação em Gerenciamento em Enfermagem da EEUSP.

${ }^{5}$ Enfermeira. Mestre em ciências pela EEUSP. 
das informações a serem extraídas dos estudos selecionados, avaliação dos estudos incluídos na revisão integrativa, interpretação dos resultados e apresentação da síntese do conhecimento.

Foi elaborada a seguinte questão norteadora: Quais são as evidências científicas nacionais e internacionais sobre a síndrome de burnout em enfermagem pediátrica e neonatal, publicadas nos últimos cinco anos?

O levantamento bibliográfico ocorreu entre os meses de novembro de 2011 a janeiro de 2012.

Os critérios de inclusão das publicações foram: textos completos publicados entre o período de janeiro de 2006 até dezembro de 2011, nas bases de Dados Medline, Cinahl, Lilacs, Dedalus e Scopus, utilizando-se os as palavras-chave: enfermagem, enfermeiros, pediatria, neonatal, neonatologia, síndrome de burnout, esgotamento profissional, nos idiomas inglês, português e espanhol. Foram encontradas 58 publicações, das quais foram selecionadas 22 estudos a partir da leitura dos resumos, identificando-se os que referiam a ocorrência da síndrome em trabalhadores de enfermagem no contexto de cuidado pediátrico ou neonatal. Excluíram-se as publicações repetidas resultando em uma amostra final de 13 estudos, sendo 12 artigos de periódicos e 1 tese de doutorado.

Após a leitura na íntegra, os dados das publicações foram transcritos para um instrumento proposto e validado na literatura e organizados em planilha eletrônica do Microsoft Excel 2007, de acordo com o ano de publicação em ordem decrescente e o título da pesquisa, permitindo maior detalhamento de cada publicação(13).

Os resultados foram apresentados em três etapas, através de quadros e sob a forma descritiva: Etapa I: organização dos dados referentes à identificação da produção (de acordo com base de dados, título, autores, ano de publicação, país e periódico; Etapa II: nível de evidência proposto pela literatura ${ }^{(14)}$; e a etapa III: fatores relacionados à ocorrência de síndrome de burnout em trabalhadores de enfermagem que assistem crianças, a partir da categorização dos mesmos.

\section{RESULTADOS}

\section{I identificação das publicações}

A amostra foi composta por 13 publicações, sendo 1 artigo publicado em periódico nacional, e as demais em outros países, com a prevalência de $8(61,5 \%)$ publicações dos EUA.

Dentre os artigos selecionados de acordo com os critérios de exclusão previamente estabelecidos, considerando a distribuição nas bases de dados selecionadas, foram encontrados 1 na base de dados Medline, 2 na Scopus, 2 na Cinahl, 1 na Lilacs, e 7 que se repetem entre as bases Medline, Scopus e Cinahl totalizando a amostra final de 13 apenas. Na Dedalus, não foi encontrado material que preenchesse os critérios de inclusão.

Quanto à aplicação de algum instrumento de avaliação da síndrome de burnout, encontramos que os estudos de n 3, 4, 8, 9 e11 aplicaram o mesmo instrumento (Maslach Burnout Inventory - MIB) na população avaliada, e utilizaram análise estatística para avaliação dos dados. Nos demais estudos encontramos utilização de outro instrumento para avaliação de sofrimento moral, estudos qualitativos ou estudos de caso, os quais também discutem a experiência de cuidado e sua relação com a síndrome de burnout, além de possíveis intervenções nesse contexto.

\begin{tabular}{|c|c|c|c|c|c|}
\hline $\begin{array}{l}\text { No do } \\
\text { estudo }\end{array}$ & $\begin{array}{c}\text { Base de } \\
\text { dados }\end{array}$ & Título & Autores & Ano/País & Periódico \\
\hline 1 & Scopus & $\begin{array}{l}\text { Coping while caring } \\
\text { for the Dying child: } \\
\text { nurses'experiences in an } \\
\text { acute care setting }\end{array}$ & $\begin{array}{l}\text { Cook, Mott, } \\
\text { Lawrence, Jablonski, } \\
\text { Grady, Norton, Liner, } \\
\text { Cioff, Hickey, Reidy, } \\
\text { Connor }\end{array}$ & 2011/EUA & Journal of Pediatric Nursing \\
\hline 2 & Cinahl & $\begin{array}{l}\text { Psychological support } \\
\text { for nurses on paediatric } \\
\text { intensive care units }\end{array}$ & Pardoe & $2011 /$ Inglaterra & $\begin{array}{l}\text { Nursing children and young } \\
\text { people }\end{array}$ \\
\hline 3 & Scopus & $\begin{array}{l}\text { Symptoms of } \\
\text { Posttraumatic Stress } \\
\text { Disorder Among Pediatric } \\
\text { Acute Care Nurses }\end{array}$ & Czaja, Moss, Mealer & 2011/EUA & Journal Pediatric Nursing \\
\hline 4 & $\begin{array}{l}\text { Cinahl, } \\
\text { Medline }\end{array}$ & $\begin{array}{l}\text { Support Programas for } \\
\text { New Graduates in Pediatric } \\
\text { Nursing }\end{array}$ & $\begin{array}{l}\text { Messmer, Bragg, } \\
\text { Williams }\end{array}$ & 2011/EUA & $\begin{array}{l}\text { The Journal of Continuing } \\
\text { Education in Nursing }\end{array}$ \\
\hline 5 & $\begin{array}{l}\text { Cinahl, } \\
\text { Medline }\end{array}$ & $\begin{array}{l}\text { Coping and Resilience } \\
\text { Factors in Pediatric } \\
\text { Oncology Nurses }\end{array}$ & Zander, Hutton, King & 2010/Austrália & $\begin{array}{c}\text { Journal of Pediatric Oncology } \\
\text { Nursing }\end{array}$ \\
\hline 6 & Medine & $\begin{array}{l}\text { How much compassion } \\
\text { have I left An exploration } \\
\text { of occupational stress } \\
\text { among children's palliative } \\
\text { care nurses }\end{array}$ & $\begin{array}{l}\text { MCCloskeu, } \\
\text { Taggart }\end{array}$ & 2010/Irlanda & $\begin{array}{l}\text { International Journal of } \\
\text { Palliative Nursing }\end{array}$ \\
\hline 7 & $\begin{array}{l}\text { Medline, } \\
\text { Cinahl }\end{array}$ & $\begin{array}{l}\text { Moral Distress in Neonatal } \\
\text { Intensive Care Unit RNs }\end{array}$ & $\begin{array}{l}\text { Cavaliere, } \\
\text { Daly, } \\
\text { Dowling, } \\
\text { Montgomer }\end{array}$ & 2010/EUA & Advances in Neonatal Care \\
\hline 8 & $\begin{array}{l}\text { Medline, } \\
\text { Cinahl }\end{array}$ & $\begin{array}{l}\text { Nurses' work } \\
\text { environments, care } \\
\text { rationing, job outcomes, } \\
\text { and quality of care on } \\
\text { neonatal units. }\end{array}$ & $\begin{array}{l}\text { Rochefort } \\
\text { Clarke }\end{array}$ & 2010/Canadá & Journal of Advanced Nursing \\
\hline
\end{tabular}




\begin{tabular}{|c|c|c|c|c|c|}
\hline 9 & Medline & $\begin{array}{l}\text { Pediatric Nurses' Grief } \\
\text { Experience: Its relationship } \\
\text { with Burnout and Job } \\
\text { Satisfaction }\end{array}$ & Adwan & 2010/EUA & Tese de Doutorado \\
\hline 10 & $\begin{array}{c}\text { Medline, } \\
\text { Scopus, } \\
\text { Cinahl }\end{array}$ & $\begin{array}{l}\text { Caring for dying children: } \\
\text { assessing the needs of the } \\
\text { pediatric palliative care } \\
\text { nurse. }\end{array}$ & Morgan & 2009/EUA/ & Pediatric Nursing \\
\hline 11 & $\begin{array}{l}\text { Cinahl, } \\
\text { Medline }\end{array}$ & $\begin{array}{l}\text { Perceptions of Stress, } \\
\text { Burnout, and support } \\
\text { systems in pediatric bone } \\
\text { marrow transplantation } \\
\text { nursing. }\end{array}$ & $\begin{array}{l}\text { Gallagher, } \\
\text { Gormley }\end{array}$ & 2009/EUA/ & $\begin{array}{c}\text { Clinical Journal of Oncology } \\
\text { Nursing }\end{array}$ \\
\hline 12 & Lilacs & $\begin{array}{l}\text { Fatores que tornam } \\
\text { estressante o trabalho de } \\
\text { médicos e enfermeiros em } \\
\text { terapia intensiva pediátrica } \\
\text { e neonatal :estudo de } \\
\text { revisão bibliográfica }\end{array}$ & $\begin{array}{l}\text { Fogaça, } \\
\text { Carvalho, } \\
\text { Cítero, } \\
\text { Nogueira-Martins }\end{array}$ & 2008/Brasil/ & $\begin{array}{c}\text { Revista Brasileira de Terapia } \\
\text { Intensiva }\end{array}$ \\
\hline 13 & $\begin{array}{l}\text { Medline, } \\
\text { Cinahl }\end{array}$ & $\begin{array}{l}\text { Nurse Burnout and Stress } \\
\text { in the NICU }\end{array}$ & Braithwaite & 2008/EUA/ & Advances in Neonatal Care \\
\hline
\end{tabular}

Quadro 1. organização dos estudos publicados acerca da síndrome de burnout em enfermagem pediátrica e neonatal, de acordo com base de dados, título, autores, ano de publicação, país e periódico. São Paulo, 2012.

\section{Nível de evidência}

Em relação ao delineamento da pesquisa, foram encontradas 2 pesquisas correlacionais (15,4\%), 2 revisões de literatura $(15,4 \%)$, 2 quantitativas (15,4\%), 1 qualitativa (7,7\%) 3 pesquisas descritivas (23,0\%) e 3 relatos de experiência (23,0\%). Dessa forma, e de acordo com a hierarquia das evidências, observa-se que 8 publicações apresentam níveis IV (61,5\%) e 3 apresentam nível V (23,0\%). De acordo com essa classificação, as revisões de literatura incluídas neste estudo não se inserem nessa hierarquia (Stetler 1998).

\section{Fatores relacionados à ocorrência da síndrome de burnout em enfermeiros que cuidam de crianças}

A partir da leitura dos materiais, surgiram três categorias temáticas a serem discutidas a seguir:

Sofrimento do profissional: os textos 1, 2, 3,7, 9, 10 e 12 reconhecem a exposição do profissional de enfermagem a situações que contribuem para a evolução para burnout: como o confronto constante com a morte e o luto, internalização de emoções, situações de dilemas éticos que entram em conflito com valores pessoais, sentimento de impotência e de sobrecarga, além de dificuldades pessoais e profissionais em estabelecer limites de envolvimento emocional com o paciente e família, e lidar com pouco diálogo entre as equipes multiprofissionais, e ameaças de pacientes ou familares.

Características Individuais: os estudos numerados 5, 6, 7,12 e 13 apontam a susceptibilidade individual como um dos fatores que contribuem para o desenvolvimento da síndrome. Fatores como idade, experiências prévias, posição ocupacional, e resiliência adicionadas ao estresse no emprego tem sido considerados fatores que prevêm burnout, além de traços de uma personalidade considerada "hardness". Sentimentos de controle nas situações da vida, uma perspectiva otimista, habilidade em lidar com mudanças e engajamento em hábitos de vida saúdaveis são traços de uma personalidade menos vulnerável ao burnout.

Suporte institucional: os trabalhos 3, 4, 5, 7, 8, 10, 11 e 13 recomendam que a instituição reconheça os estressores do ambiente na tentativa de estabelecer medidas que possam amenizar o sofrimento ou oferecer estratégias de apoio à equipe, considerando-se também necessidade de suporte às enfermeiras recém-formadas, no que diz respeito a iniciar uma nova profissão, lidar com pacientes difíceis e seus familiares, e orientação para uso de equipamentos. São sugeridas também discussões de dilemas éticos entre a equipe multiprofissional.

\section{DISCUSSÃO DOS RESULTADOS E SÍNTESE DAS EVIDÊNCIAS}

Observa-se que a maior parte dos artigos foi localizada entre as bases de dados Medline e Cinahl. A Medline é uma base de dados especializada na literatura médica e biomédica de caráter internacional, enquanto que a Cinahl é o recurso mais abrangente para a enfermagem e literatura na área de saúde com textos na íntegra e um grande número de revistas indexadas.

Quanto ao local de publicação e idioma, observa-se grande concentração das publicações em língua inglesa e nos EUA, sendo este o local onde se originou o termo burnout.

Nesta presente revisão, foram constatados 8 artigos com nível de evidência IV e 3 com nível de evidência $V$, além de 2 revisões de literatura. Apenas os estudos com níveis de evidência I e II (metanálise e ensaio clínico randomizado controlado) possuem recomendações de condutas. No entanto, essa temática, embora existam instrumentos de mensuração, como o Maslach Burnout Inventory, talvez não tenha sido ainda devidamente abordada no contexto de cuidado à criança, embora seja reconhecida aqui neste estudo a vulnerabilidade do profissional de enfermagem.

A partir dos dados apresentados, observamos que as adversidades no modo como ocorre o processo de trabalho, possibilitam o desequilíbrio na relação saúde-doença. Os resultados dessa revisão estão de acordo com estudos que reforçam relação significativa da ocorrência da síndrome com questões como sobrecarga de trabalho, ausência de coleguismo, falta de suporte institucional e o sofrimento produzido pela angústia que perpassa às situações do trabalho, entre outras questões ${ }^{(6,15-17)}$.

Quanto à percepção de necessidade de suporte institucional, observa-se que a síndrome de burnout se relaciona mais fortemente com os aspectos do ambiente de trabalho do que com características individuais dos trabalhadores, embora seja reconhecido que ela resulta da dinâmica entre o ambiente e o indivíduo.

Nesse pensamento, enfatiza-se a importância de um ambiente social acolhedor no controle do estresse emocional, colocando a organização como interveniente entre essas variáveis. A literatura aponta ainda a alta correlação encontrada entre o fator estilo de gestão da chefia e o fator exaustão emocional ${ }^{(9,18)}$. 


\section{CONCLUSÕES}

Foram analisados 13 estudos acerca da síndrome de burnout em trabalhadores de enfermagem no contexto de cuidado pediátrico ou neonatal, sendo 1 artigo publicado em periódico nacional, e as demais em outros países, como EUA, Canadá, Austrália, Irlanda e Inglaterra, com grande parte dos estudos extraídos de publicações de enfermagem e com predomínio do idioma inglês. Quanto à aplicação de algum instrumento de avaliação da síndrome de burnout, encontramos 5 estudos que aplicaram o mesmo instrumento (Maslach Burnout Inventory-MIB) na população avaliada, e utilizaram análise estatística para avaliação dos dados, sendo que os demais desenvolveram estudo qualitativo, de revisão, ou com aplicação de outro tipo de instrumento. As publicações avaliadas apresentam níveis de evidência IV ou nível V.

No que diz respeito à categorização das informações acerca dos fatores relacionados à ocorrência da síndrome de burnout em enfermeiros que cuidam de crianças, surgiram as seguintes categorias: Sofrimento do profissional, Características Individuais e Suporte institucional. Tais informações revelam a relação entre os vários aspectos do trabalho de enfermagem que tornam o profissional dessa categoria mais vulnerável ao burnout, além da a relação estreita entre os construtos percepção de suporte da organização e a ocorrência da síndrome de burnout.

Diante dos estudos analisados, sugerem-se mais estudos no campo de atuação da enfermagem pediátrica, inclusive em cenário brasileiro, com níveis de evidência fortes para subsidiar intervenções no que diz respeito à saúde do trabalhador de enfermagem, uma vez que, nessa revisão não foi encontrado nenhum trabalho com essa metodologia e no Brasil foi encontrada apenas uma revisão de literatura acerca do tema.

\section{Referências}

1. Trindade LL, Lautert L. Síndrome de Burnout entre os trabalhadores da estratégia de Saúde da Família. Rev Esc Enferm USP. 2010; 44(2): 274-279.

2. Jodas DA, Haddad MCL 2009. A síndrome de Burnout em trabalhadores de enfermagem de um pronto socorro de Hospital Universitário. Acta Paul Enferm. 2009; 22(2): 192-197

3. Grazziano ES. Estratégia para redução do stress e burnout entre enfermeiros hospitalares. [Tese]. São Paulo; Escola de Enfermagem da Universidade de São Paulo; 2008; 232p.

4. Moreira DS, Magnago RF, Sakae TM, Magajewski FRL 2009. Prevalência da síndrome de burnout em trabalhadores de enfermagem de um hospital de grande porte da Região Sul do Brasil. Cad. Saúde Pública, Rio de Janeiro. 2009; 25(7): 1559-1568.

5. Manetti ML, Marzialle MHP. Fatores associados à depressão relacionada ao trabalho de enfermagem. Estudos de Psicologia. 2007, 12(1), 79-85.

6. Pereira AMTB. Burnout: quando o trabalho ameaça o bem-estar do trabalhador. São Paulo: Casa do Psicólogo; 2010.

7. Fogaça MC, Carvalho WB, Cítero VA, Nogueira-Martins LA Fatores que tornam estressante 0 trabalho de médicos e enfermeiros em terapia intensiva pediátrica e neonatal: estudo de revisão bibliográfica. Rev Bras Ter Intensiva. 2008; 20(3): 261266.

8. Guerrer FJL, Bianchi ERF. Caracterização do estresse nos enfermeiros de unidades de terapia intensiva. Rev Esc Enferm USP 2008; 42(2): 355-62.

9. Oliveira PR, Tristão MR, Neiva ER. Burnout e suporte organizacional em profissionais de UTI-Neonatal. Edu Pro: C \&
T.2006; 1(1), 27-37

10. Ganong LH. Integrative reviews of nursing research. Res Nurs Health. 1987;10(1):1-11

11. Whittemore $R$, Knafl $K$. The integrative review: update methodology. J Adv Nurs. 2005; 52(5): 546-53.

12. Melo MB, Barbosa MA, Souza PR. Satisfação no trabalho da equipe de enfermagem: revisão integrativa. Rev. Latino-Am. Enfermagem jul/ago. 2011; 19(4).

13. Ursi ES. Prevenção de lesão de pele no perioperatório: uma revisão integrativa da literatura [dissertação]. Ribeirão Preto: Escola de Enfermagem de Ribeirão Preto/ Universidade de São Paulo; 2005. $128 \mathrm{p}$.

14. Stetler CB, Morsi D, Rucki S, Broughton S, Corrigan B, Fitzgerald $\mathrm{J}$, et al. Utilization-Focused Integrative Reviews in a Nursing Service. Applied Nurs Res. 1998;11(4):195- 206.

15. Ezaias GM, Gouvea PB, Haddad MCL, Vannuchi MTO, Sardinha DSS. Síndrome de burnout em trabalhadores de saúde em um hospital de média complexidade. Rev. enferm. UERJ. 2010; 18(4): 524-9.

16. Tamayo MR. Burnout: Implicações das Fontes Organizacionais de Desajuste Indivíduo-Trabalho em Profissionais da Enfermagem. Psicologia: Reflexão e Crítica. 2009; 22(3): 474-482.

17. Feliciano KVO, Kovacs MH, Sarinho SW. Sentimentos de profissionais dos serviços de pronto-socorro pediátrico: reflexões sobre o burnout. Rev. Brasileira de Saúde Materno Infantil. 2005; 5(3). 18. Souza WC, Silva AGMM 2002. A influência de fatores de personalidade e de organização do trabalho no burnout em profissionais de saúde. Rev. Estudos de Psicologia, PUC-Campinas. 2002; 19(1): p. 37-48. 